\title{
Surgical Treatment Strategy for Distal Humerus Intra-articular Fractures
}

\author{
Hyo-Jin Lee \\ Department of Orthopedic Surgery, Seoul St. Mary's Hospital, College of Medicine, The Catholic University of Korea, Seoul, Korea
}

\begin{abstract}
Treating distal humerus fractures, especially those involving intra-articular lesions, is complex and often technically demanding. Although there still exist many controversial issues, the goal of treatment is to establish anatomical stable fixation by restoring the two columns and the articular surface. Universally, a posterior midline incision is applied, and the approach varies according to the further management of the triceps or olecranon. Evidence supports dual plate fixation as the optimal fixation method, and debates regarding appropriate plating configuration are still ongoing. As multiple clinical studies comparing results of parallel and perpendicular plate fixation have shown no actual difference, it is important to place the plates according to the fracture configuration.
\end{abstract}

(Clin Shoulder Elbow 2019;22(2):113-117)

Key Words: Humerus; Intra-articular fractures; Fracture fixation

\section{Introduction}

Fractures of the distal humerus in adults are considered to be challenging due to the combination of anatomic and treatment complexities, poor outcomes, and complications. The estimated incidence in adults is 5.7 per 100,000 people every year, representing less than $7 \%$ of adult fractures. ${ }^{1,2)}$ These fractures have a bimodal distribution, with an early peak in young males due to high-energy trauma, and a late peak in the elderly due to lowenergy trauma.

Distal humerus fractures are commonly classified by the Orthopaedic Trauma Association/Arbeitsgemeinschaft für Osteosynthesefragen (OTA/AO) classification. Fractures are categorized into three types: type A designates an extra-articular fracture, type $B$ designates a partial articular fracture, and type $C$ designates a complete articular fracture in which the articular surface is completely dissociated from the humeral shaft.

Despite great advancements in the treatment of distal humerus fractures, there exist numerous controversial issues, including the optimal surgical approach, fixation method, implants, and management of the ulnar nerve. This article focuses on the strategy for open reduction and internal fixation of distal humerus intra-articular fractures.

\section{Preoperative Plan}

A complete review of history, including injury mechanism and preinjury medical condition, needs to be analyzed. The condition of the skin or the existence of open wounds should be carefully observed. Nerve function (especially ulnar and radial nerve) and vascular conditions, including peripheral pulse, should be evaluated prior to surgical intervention. For imaging studies, plain radiography is usually sufficient for diagnosis and establishing a treatment plan. Computed tomography scanning is helpful in understanding the fracture pattern, especially when coronal plane injuries such as shear fracture of the capitellum and trochlea are suspected.

Received December 14, 2018. Revised March 1, 2019. Accepted April 7, 2019.

Correspondence to: Hyo-Jin Lee

Department of Orthopedic Surgery, The Catholic University of Korea, Seoul St. Mary's Hospital, 222 Banpo-daero, Seocho-gu, Seoul 06591, Korea

Tel: +82-2-2258-6121, Fax: +82-2-535-9834, E-mail: hyojin1229@gmail.com, ORCID: https://orcid.org/0000-0002-7708-4754

Review article does not need an IRB approval.

Financial support: None. Conflict of interests: None. 


\section{Basic Settings}

Surgical treatment for a distal humerus fracture often requires prolonged surgery. The prone or lateral decubitus position is recommended for good visualization of the lesion and because it allows easy positioning of the C-arm. All pressure points must be carefully padded to avoid nerve compression and sore formation due to prolonged operation time.

A universal posterior midline incision is made when the fracture involves the condyle and the surrounding region. Injecting Marcaine with epinephrine helps with hemostasis during the exposure. Full-thickness medial and lateral fasciocutaneous flaps are raised to avoid devascularization of the skin and seroma formation. In case of olecranon osteotomy, the incision should curve around the lateral aspect of the olecranon to facilitate hardware coverage, and to avoid direct irritation which may result in hematoma formation or skin problems in future.

\section{Management of the Ulnar Nerve}

Since it is important to identify the ulnar nerve along the medial border of the triceps muscle, the deep fascia is elevated for easy identification (Fig. 1). For complete mobilization, the nerve is dissected from its proximal to distal end. For the proximal portion, the arcade of Struther, which is found $8 \mathrm{~cm}$ proximal to the epicondyle, is released. The nerve is traced distally 6 to $8 \mathrm{~cm}$, and the cubital tunnel retinaculum is released. The motor branches to the flexor carpi ulnaris and the flexor digitorum profundus are preserved. There is no consensus regarding the management of the released ulnar nerve, whether to maintain it in situ or transpose it anteriorly. A recent meta-analysis reported an overall neuropathy incidence of $15.3 \%$ in the release only

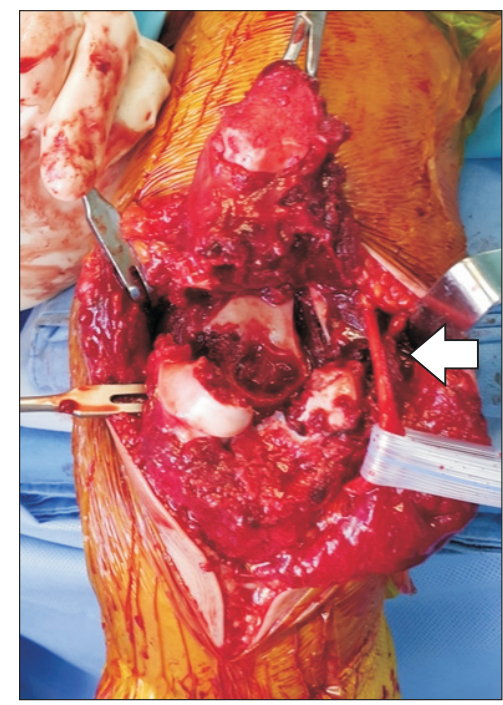

Fig. 1. Identification of the ulnar nerve (arrow). group, and $23 \%$ in the transposition group. ${ }^{3)}$ They concluded that transposition of the ulnar nerve exerts no protective effect. However, prior transposition of the ulnar nerve before fixation may have an advantage for selecting the plate position on the medial side.

\section{Operative Approaches}

\section{Paratricipital Approach}

Dissection is continued to the medial and lateral triceps border. The triceps muscle is separated from the posterior surface of the intermuscular septum. On the lateral aspect, the radial nerve is identified passing from the posterior to the anterior direction through the intermuscular septum. After elevating the triceps muscle from the periosteum and reflecting it medially, the posterolateral humerus shaft is exposed. The anconeus muscle is then elevated in conjunction with the triceps to preserve its innervation and blood supply. For the medial exposure, the ulnar nerve is first identified and exposed. Medial dissection along the posterior border of the intermuscular septum exposes the posteromedial aspect of the distal humerus. Visualization of the entire posterior humerus is possible after mobilization and elevation of the triceps from the posterior cortex of the humerus. Since this approach spares the olecranon and extensor mechanism and avoids an olecranon osteotomy, the surgical time is shortened, thereby reducing the risks of perioperative or postoperative complications. ${ }^{4-6)}$ The approach is generally adequate for extra-articular fracture and permits an articular reduction of the C1 and C2 fracture patterns with large medial and epicondylar fragments. However, in the case of an intraarticular multifragmentary (C3) fracture, it requires further exposure through an olecranon osteotomy. This approach can be converted to a more extensile exposure, with or without an olecranon osteotomy, during the procedure."

\section{Triceps-reflecting Approach (Bryan-Morrey)}

The importance of this approach is that the triceps tendon, forearm fascia, and periosteum are released as one unit from medial to lateral as a continuous sleeve, reflected directly off the olecranon. Proximally, the entire extensor mechanism and posterior capsule are reflected and retracted laterally to expose the joint. As the triceps are retracted laterally, careful monitoring of the ulnar nerve is required to avoid a traction injury. This approach was originally introduced for arthroplasty. After fracture fixation, the triceps tendon is reattached to the olecranon by drilling two holes in the cruciate configuration. Also, the patient is required to avoid active elbow extension with resistance for six weeks. As long as the extensor mechanisms are tightly secured, there will be no disruption of the extensor mechanism. However, due to the pull of the common extensor muscles and the lateral reflection of the triceps, access to the lateral epicondyle 
has always been challenging. ${ }^{8,9)}$

\section{Triceps-reflecting Anconeus Pedicle Flap}

The triceps-reflecting anconeus pedicle flap (TRAP) approach, described by $\mathrm{O}^{\prime}$ Driscoll, ${ }^{10)}$ is somewhat similar to the BryanMorrey approach, with the triceps reflected off the olecranon in continuity with the anconeus. The difference is that in the TRAP approach, a V-shaped incision is made to reflect the triceps and anconeus completely off the triceps. The anconeus muscle is fully released from insertion on the proximal lateral ulna. This approach guarantees adequate visualization of the articular surface with no significant difference from the trans-olecranon approach. Also, it preserves the olecranon and can be converted to arthroplasty when needed. After the procedure, the repair is made through drill holes in the olecranon.

\section{Triceps-splitting Approach}

The triceps-splitting approach, ${ }^{11,12)}$ also known as the Campbell approach, involves a midline incision in the triceps, and divides the triceps into equal medial and lateral halves. The incision continues to the olecranon insertion, and the triceps are peeled subperiosteally to expose the distal humerus. Proximally, the radial nerve needs to be carefully protected during the exposure. Even though this approach is suitable for distal diaphyseal fractures, it does not provide proper exposure of the distal articular surface. ${ }^{13)}$ Furthermore, it may result in a negative effect on muscle strength due to direct muscle injury with resultant fibrosis and injury to the intramuscular nerve branches.

\section{Olecranon Osteotomy}

As compared to the other approaches discussed above, the olecranon osteotomy $^{14)}$ approach provides superior visualization of the articular surface of the distal humerus. ${ }^{13)}$ An apex distal, chevron-type osteotomy is made at 2.5 to $3 \mathrm{~cm}$ from the tip of the olecranon. In order to avoid further damage to the articular surface, we recommend using an osteotome for the completion of the osteotomy after using an oscillating saw (Fig. 2). On completion of the procedure, the osteotomy site is fixed with tension band wires, an intramedullary screw or a plate. The major complications include hardware prominence and delayed union or nonunion.

\section{Fixation Strategy}

The primary principle for surgical fixation of a distal humerus fracture includes anatomic articular reduction and rigid fixation with dual plates. Anatomic reduction of the articular surface together with restoration of the humerus alignment should be obtained by surgical treatment. Even though clear exposure and visualization of the fracture lesion are essential for successful fixation, great care should be taken to avoid disrupting the remaining soft tissue attachments.
According to the guidelines proposed by Arbeitsgemeinschaft für Osteosynthesefragen/Association for the Study of Internal Fixation (AO/ASIF), articular fragments should be reduced first. Depending on the fixation plan, these articular fragments can be fixed using separate screws, or provisionally fixed using Kirschner wires. If there is no comminution in the metaphysis, creating a stable platform by prior reduction of the metaphyseal column may help reduction of the articular surface. During reconstruction of the articular surface, lag screws are often placed either through or outside a plate to cross the entire capitellum and trochlea. Sanchez-Sotelo et al. ${ }^{15)}$ recommended that every screw in the distal fragment should pass through a plate. Plates should be placed on each column to augment the screw fixation. Also, their principles for fracture stabilization are to maximize fixation in the distal fragments and to maximize fracture stability at the supracondylar level.

Once the articular surface has been reduced, the next step requires compression of the articular surface to the metaphysis or distal humeral shaft, followed by plate fixation. Ideally, plates should be applied after adequate reduction of the fracture. However, the indirect reduction technique using plates can be helpful in some type $\mathrm{C}$ fractures. Plate fixation provides the rigidity required to optimize the union of the fracture. In case of a type $\mathrm{C}$ fracture, fixation relying merely on screw fixation is unacceptable under any circumstance. Various constructs have been proposed to fix the articular surface to the proximal portion. Different types of plates were introduced for fixation, ranging from the $3.5 \mathrm{~mm}$ reconstruction plate to anatomically contoured plates, with both locking and non-locking options. Among the many plates introduced, one-third tubular plates were determined to be too weak to endure secure bone fixation. ${ }^{16)}$

While dual plate fixation is the essential principle in the treat-

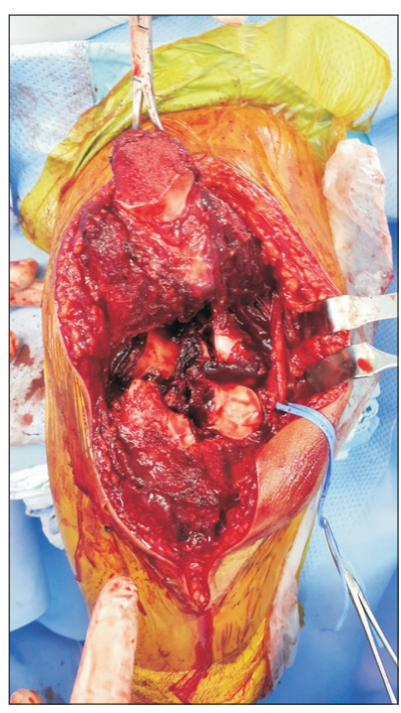

Fig. 2. Olecranon osteotomy. 
ment of a distal humerus fracture, there is considerable debate regarding the most appropriate orientation or position of the fixation (Fig. 3). According to the guidelines proposed by AO/ASIF, the two plates should be applied at a 90-degree angle to one another after the initial fixation of the articular fragments with screws. ${ }^{14,17,18)}$ However, although this technique guarantees adequate distal fixation, insufficient stability between the distal and proximal fragments could result in nonunion. ${ }^{19)}$ Furthermore, prolonged immobilization to prevent failure due to insufficient fixation can lead to joint stiffness. ${ }^{20)}$

New objectives have been proposed to increase the stability by enhancing fixation of the distal fragments and adding distal segment fixation to fracture stability at the supracondylar level. ${ }^{15)}$ In order to achieve this, several long screws are used to lock together by interdigitation at the distal segment. Also, these screws require to be attached to the plate on one side and into a bone fragment on the other side, and are not fixed on their own separately. However, it is not easy to place multiple screws distally due to a high chance of collision during the insertion. To overcome this problem, a locking plate with a fixed screw angle should be avoided, and an oscillating mode and not drilling mode, is recommended during the process of drilling for the screw.

While there has been a clear consensus on applying dual plates as a treatment option, the debate regarding the most appropriate position of the plates is still ongoing. This debate mostly involves whether the plates should be fixed in a parallel fashion or perpendicular to each other (Fig. 3). Many biomechanical comparisons have been attempted, simulating metaphyseal comminution by establishing a gap model. Several biomechanical studies have demonstrated that parallel plate fixation is substantially more stable than perpendicular plating. ${ }^{18,21-23)}$ However, a recent prospective randomized study comparing the clinical results of perpendicular and parallel plating found no differences
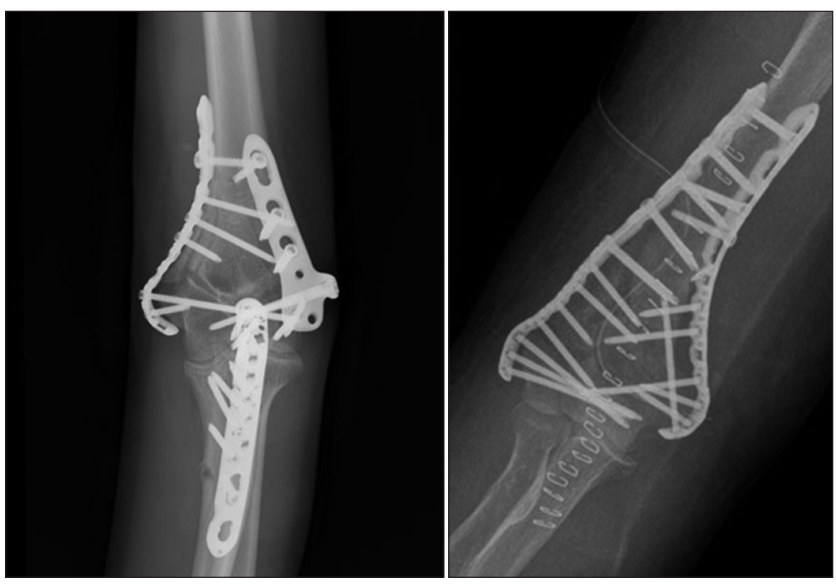

Fig. 3. Perpendicular plating and parallel plating for fixation of distal humerus fracture. in terms of clinical outcomes or complication rates. ${ }^{24)}$ Similar results have been reported by Shin et al., ${ }^{25)}$ showing no significant clinical differences. However, they reported two nonunions in the perpendicular plating group, and no nonunion in the parallel plating group. With no actual differences in clinical outcomes, it is important to place the plates according to the fracture configuration. For example, if the trochlea is a separate fragment from the rest of the medial epicondyle, the medial plates should wrap around the medial epicondyle with the screws across the articular components through the plate. ${ }^{16)}$ Parallel plating is preferred for the fixation of fractures involving the most distal end of the humerus, whereas perpendicular plating is preferred in cases of coronal shear fractures, where posterior to anterior fixation may provide additional stability. ${ }^{24)}$

Despite all the controversies, the key to achieving stable fixation is an excellent reduction of the fracture itself. Without successful construction of the triangular construct of the distal humerus, which is represented by bony medial and lateral columns and linking of the articular segment, firm anatomical fixation cannot be executed.

\section{Conclusion}

Decision regarding the treatment for intra-articular fractures of the distal humerus should be based on a combination of the best available evidence and preference of the surgeon. Given that these principles are followed during the procedure, the overall outcomes of treatment for intra-articular fracture are generally quite satisfactory. Also, early stability achieved by surgical intervention can lead to superior results by early intensive rehabilitation to restore elbow motion.

\section{References}

1. Robinson CM, Hill RM, Jacobs N, Dall G, Court-Brown CM. Adult distal humeral metaphyseal fractures: epidemiology and results of treatment. J Orthop Trauma. 2003;17(1):38-47. doi: 10.1097/00005131-200301000-00006.

2. Galano GJ, Ahmad CS, Levine WN. Current treatment strategies for bicolumnar distal humerus fractures. J Am Acad Orthop Surg. 2010;18(1):20-30. doi: 10.5435/00124635$201001000-00004$.

3. Shearin JW, Chapman TR, Miller A, llyas AM. Ulnar nerve management with distal humerus fracture fixation: a metaanalysis. Hand Clin. 2018;34(1):97-103. doi: 10.1016/j.hcl. 2017.09.010.

4. Gabel GT, Hanson G, Bennett JB, Noble PC, Tullos HS. Intraarticular fractures of the distal humerus in the adult. Clin Orthop Relat Res. 1987;(216):99-108. doi: 10.1097/00003086$198703000-00016$.

5. Gainor BJ, Moussa F, Schott T. Healing rate of transverse oste- 
otomies of the olecranon used in reconstruction of distal humerus fractures. J South Orthop Assoc. 1995;4(4):263-8.

6. Zagorski JB, Jennings JJ, Burkhalter WE, Uribe JW. Comminuted intraarticular fractures of the distal humeral condyles. Surgical vs. nonsurgical treatment. Clin Orthop Relat Res. 1986;(202):197-204. doi: 10.1097/00003086-19860100000026.

7. Schildhauer TA, Nork SE, Mills WJ, Henley MB. Extensor mechanism-sparing paratricipital posterior approach to the distal humerus. J Orthop Trauma. 2003;17(5):374-8. doi: 10.1097/00005131-200305000-00009.

8. Bryan RS, Morrey BF. Extensive posterior exposure of the elbow: a triceps-sparing approach. Clin Orthop Relat Res. 1982;(166):188-92. doi: 10.1097/00003086-19820600000033.

9. Ek ET, Goldwasser M, Bonomo AL. Functional outcome of complex intercondylar fractures of the distal humerus treated through a triceps-sparing approach. J Shoulder Elbow Surg. 2008;17(3):441-6. doi: 10.1016/j.jse.2007.08.012.

10. O'Driscoll SW. The triceps-reflecting anconeus pedicle (TRAP) approach for distal humeral fractures and nonunions. Orthop Clin North Am. 2000;31(1):91-101. doi: 10.1016/S00305898(05)70130-9.

11. Anglen J. Distal humerus fractures. J Am Acad Orthop Surg. 2005;13(5):291-7.

12. Ziran BH, Smith WR, Balk ML, Manning CM, Agudelo JF. A true triceps-splitting approach for treatment of distal humerus fractures: a preliminary report. J Trauma. 2005;58(1):70-5. doi: 10.1097/01.TA.0000145079.76335.DD.

13. Wilkinson JM, Stanley D. Posterior surgical approaches to the elbow: a comparative anatomic study. J Shoulder Elbow Surg. 2001;10(4):380-2. doi: 10.1067/mse.2001.116517.

14. Jupiter JB, Neff $U$, Holzach P, Allgöwer M. Intercondylar fractures of the humerus. An operative approach. J Bone Joint Surg Am. 1985;67(2):226-39. doi: 10.2106/00004623198567020-00008.

15. Sanchez-Sotelo J, Torchia ME, O'Driscoll SW. Complex distal humeral fractures: internal fixation with a principle-based par- allel-plate technique. J Bone Joint Surg Am. 2007;89(5):961-9. doi: 10.2106/JBJS.E.01311.

16. Miller AN, Beingessner DM. Intra-articular distal humerus fractures. Orthop Clin North Am. 2013;44(1):35-45. doi: 10.1016/j.ocl.2012.08.010.

17. Helfet DL, Schmeling GJ. Bicondylar intraarticular fractures of the distal humerus in adults. Clin Orthop Relat Res. 1993;(292):26-36. doi: 10.1097/00003086-19930700000006.

18. Self J, Viegas SF, Buford WL Jr, Patterson RM. A comparison of double-plate fixation methods for complex distal humerus fractures. J Shoulder Elbow Surg. 1995;4(1 Pt 1):10-6.

19. Ackerman G, Jupiter JB. Non-union of fractures of the distal end of the humerus. J Bone Joint Surg Am. 1988;70(1):75-83.

20. Ring D, Jupiter JB. Fractures of the distal humerus. Orthop Clin North Am. 2000;31(1):103-13.

21. Schemitsch EH, Tencer AF, Henley MB. Biomechanical evaluation of methods of internal fixation of the distal humerus. J Orthop Trauma. 1994;8(6):468-75. doi: 10.1097/00005131199408060-00003.

22. Stoffel K, Cunneen S, Morgan R, Nicholls R, Stachowiak G. Comparative stability of perpendicular versus parallel doublelocking plating systems in osteoporotic comminuted distal humerus fractures. J Orthop Res. 2008;26(6):778-84. doi: 10.1002/jor.20528.

23. Arnander MW, Reeves A, MacLeod IA, Pinto TM, Khaleel A. A biomechanical comparison of plate configuration in distal humerus fractures. J Orthop Trauma. 2008;22(5):332-6. doi: 10.1097/BOT.0b013e31816edbce.

24. Lee SK, Kim KJ, Park KH, Choy WS. A comparison between orthogonal and parallel plating methods for distal humerus fractures: a prospective randomized trial. Eur J Orthop Surg Traumatol. 2014;24(7):1123-31. doi: 10.1007/s00590-0131286-y.

25. Shin SJ, Sohn HS, Do NH. A clinical comparison of two different double plating methods for intraarticular distal humerus fractures. J Shoulder Elbow Surg. 2010;19(1):2-9. doi: 10.1016/j.jse.2009.05.003. 